\title{
Tuberculosis en niños y adolescentes: estrategias para la intervención de los trabajadores sociales
}

\author{
Tuberculosis in children and adolescents: Strategies for social \\ workers' interventions
}

Dra. Norma E. González $z^{a}$ y Lic. Luciana Angueira ${ }^{b}$

\section{RESUMEN}

En la asistencia de niños y adolescentes enfermos de tuberculosis, se deben conocer las dificultades que tienen muchas familias para acceder a la atención médica y al diagnóstico y tratamiento oportuno. El trabajador social actúa juntoconotros integrantes del equipo de salud, lo que facilita el acceso a los recursos y beneficios asistenciales que pueden favorecer la adherencia al tratamiento y fortalecer la salud de esa población vulnerable. Si bien el propósito que engloba el accionar del trabajador social en esta enfermedad es contribuir a disminuir el riesgo de infectarse, enfermar o morir detuberculosis, la situación epidemiológica actual de esta patología en Argentina ha puesto al trabajador social frente al desafío de replantearse nuevas estrategias de intervención y revisión de objetivos. El objetivo de este trabajo es plantear su rol y proponer acciones que pueden contribuir a disminuir la morbimortalidad por tuberculosis en niños y adolescentes.

Palabras clave: tuberculosis, trabajo social, niños.

a. División

Neumotisiología, Hospital de Niños Dr. Pedro de Elizalde, Ciudad Autónoma de Buenos Aires, Argentina.

b. Programa Nacional de Control de la Tuberculosis y Lepra. Red de Trabajadoras Sociales que intervienen en Tuberculosis del área metropolitana de Buenos Aires.

\section{Correspondencia:}

Dra. Norma E. González: negonza@intramed.net.

Financiamiento:

Ninguno.

Conflicto de intereses:

Ninguno que declarar.

Recibido: 11-2-2017

Aceptado: 22-5-2017

\section{INTRODUCCIÓN}

La tuberculosis en Argentina continúa siendo una deuda pendiente de los sistemas de salud, que no han podido evitar que niños y adolescentes sigan muriendo debido a esta enfermedad. La Organización Mundial de la Salud estima que hay 9 millones de enfermos y 1 millón y medio de muertes por tuberculosis en el mundo cada año; esto la convierte en una de las enfermedades infecciosas más letales. ${ }^{1}$

A pesar de contar con medidas sanitarias eficientes para poder controlarla, en nuestro país, se producen 10 mil casos por año, 70\% en personas en edad productiva y más de la mitad en bacilíferos (pacientes que eliminan el bacilo tuberculoso en sus secreciones respiratorias), fuente de transmisión activa de la enfermedad a la población sana. ${ }^{2}$ El número de enfermos pulmonares bacilíferos registrados en los últimos años no muestra un ritmo de reducción suficiente como para que el riesgo de exposición al contagio de la población pediátrica disminuya. ${ }^{2}$

Según el Instituto Nacional de Enfermedades Respiratorias "Emilio Coni", en 2015, se notificaron, en Argentina, 10713 casos de tuberculosis (24,8 casos / 100000 habitantes); 9,6\% eran niños menores de 15 años y 9,3\%, adolescentes de entre 15 y 19 años. ${ }^{2}$

Entre 2011 y 2015, se registraron 117 defunciones por tuberculosis en menores de 20 años, lo que representó una tasa ajustada por edad de 0,16 por 100000 habitantes. En el grupo de 15 a 19 años, la tasa fue 1,65 veces mayor que en el grupo de 10 a 14 años y más 
de 5 veces superior que en el grupo de menores de 10 años. ${ }^{3}$

El diagnóstico en edades pediátricas es más difícil y la confirmación microbiológica, poco frecuente. Por este motivo, es probable que haya un subregistro de casos pediátricos. Muchos niños que fallecen con complicaciones de meningitis, neumonía o desnutrición pueden ser enfermos de tuberculosis que no llegan a ser notificados como tales a los programas de control. ${ }^{4}$

Además, en nuestro país, el análisis de los indicadores epidemiológicos registrados entre 1990 y 2011 muestra una distribución desigual de los casos de tuberculosis en las distintas jurisdicciones, y se asocian las mayores tasas de enfermedad a condiciones sociales desfavorables de la población. ${ }^{5}$

La calidad de atención brindada a los pacientes varía en cada lugar según los recursos disponibles. Equipar las áreas de mayor concentración de casos con los elementos necesarios para la prevención, diagnóstico precoz $\mathrm{y}$ tratamiento completo gratuito no es suficiente para lograr reducir las tasas de enfermedad ${ }^{6-9} \mathrm{En}$ el proceso de atención de los niños y adolescentes con tuberculosis, también se deben conocer las dificultades que tienen muchas familias para acceder al diagnóstico y tratamiento oportuno, así como a los recursos y beneficios asistenciales que pueden favorecer la adherencia al tratamiento y fortalecer la salud de esa población vulnerable. ${ }^{10}$

Históricamente, los avances en la medicina y el desarrollo social y económico han contribuido a disminuir la prevalencia de tuberculosis en muchos países. ${ }^{7}$ Sin embargo, la aparición del virus de inmunodeficiencia humana y el síndrome de inmunodeficiencia adquirida (VIH/sida) y de multirresistencia a fármacos han puesto en evidencia que los factores por considerar para controlar y erradicar la tuberculosis son mucho más complejos y deben abordarse con una visión más amplia. ${ }^{4,10-12}$

Existen publicaciones de organismos internacionales ${ }^{10,13}$ y grupos de trabajo ${ }^{6,9}$ que plantean oportunidades de intervención en el aspecto social de la tuberculosis. El abordaje multidisciplinario contribuye a mejorar la calidad de atención de los enfermos, pero el resultado depende, fundamentalmente, de las actitudes, habilidades y competencias del personal de salud. ${ }^{13}$

Luego de haber participado en varias instancias de capacitación en tuberculosis infantil realizadas en diferentes provincias de Argentina (en el marco de la iniciativa Cero Muertes por Tuberculosis de la Sociedad Argentina de Pediatría y el Ministerio de Salud de la Nación) y desde nuestro trabajo asistencial, en un centro de referencia pediátrico y otro de atención primaria del área metropolitana de Buenos Aires, nos surge la necesidad de enfocar este aspecto.

Nuestro objetivo es plantear el rol del trabajador social en el manejo de la tuberculosis en niños y adolescentes, y proponer actividades de intervención que puedan contribuir a disminuir la morbimortalidad por dicha enfermedad en esta población.

\section{Abordaje del problema de la tuberculosis}

El trabajador social actúa en áreas asistenciales junto con otros integrantes del equipo de salud. $\mathrm{Su}$ actividad se encuadra en concepciones referidas al proceso salud-enfermedad-atención, al derecho a la asistencia sanitaria de todos, lo que facilita la accesibilidad a su diagnóstico y tratamiento.

Si bien el propósito que engloba el accionar del trabajador social en esta enfermedad es contribuir a disminuir el riesgo de infectarse, enfermar o morir de tuberculosis, la actual demanda de esta patología, tanto en consultorios externos como en salas de internación, ha puesto al trabajador social frente al desafío de replantearse nuevas estrategias de intervención y revisión de objetivos. ${ }^{14}$

Tradicionalmente y hasta la actualidad, en muchas ocasiones, las personas enfermas son consideradas meros receptores de indicaciones médicas, lo que convierte al paciente en un sujeto pasivo. Se entiende que su encuentro con el equipo de salud debe estar basado en una escucha atenta y respetuosa, en la que el paciente sea protagonista de su tratamiento y se apropie conscientemente del cuidado de su salud, para convertirlo en un sujeto activo que pueda expresar sus dudas, sus temores, sus conocimientos. En el caso de lactantes y niños pequeños, la madre, el padre o el familiar a cargo, muchas veces, pueden manifestar sus inquietudes sobre el diagnóstico y tratamiento con más confianza ante un trabajador social que en el momento de la consulta con el pediatra.

Esto no invalida la responsabilidad de los otros profesionales de la salud, sino que inscribe su intervención en una lógica diferente y da lugar a las condiciones de vida de cada sujeto, sus experiencias y representaciones sobre la enfermedad..$^{15}$ Se hace referencia a la 
particular inserción que cada adolescente, niño o adulto a cargo de su cuidado tiene en el ámbito familiar, escolar, relacional y a la aceptación de la enfermedad.

Al plantear las acciones por tomar, se debe tener en cuenta que se trata de una enfermedad infectocontagiosa, por lo cual la atención incluye no solo a la persona enferma, sino también a sus convivientes y a quienes hayan permanecido largo tiempo con ella, a los que se denomina "contactos". Los que viven bajo el mismo techo del enfermo bacilífero tienen más riesgo de adquirir la infección. El riesgo de contagio de los contactos pediátricos es mayor que el de los adultos, especialmente, en los lactantes y niños pequeños.

Por ser una enfermedad "contagiosa" y ser considerada "una enfermedad de la pobreza", ${ }_{1}$ la estigmatización y marginación de quienes la padecen puede ser una barrera para aceptar el diagnóstico ${ }^{16} \mathrm{y}$, mucho más, comunicarlo a sus familiares para que asistan a los centros de salud a realizar los exámenes diagnósticos necesarios. ${ }^{6}$

Los niños pequeños, generalmente, no contagian, pero los niños mayores y adolescentes pueden tener una forma de tuberculosis similar a la que presentan los adultos, es decir, también pueden ser enfermos bacilíferos. Estos pacientes, en las semanas previas al diagnóstico, suelen haber estado asistiendo a instituciones educativas donde pasan varias horas al día y exponen a sus compañeros de curso y docentes a adquirir la infección. Por esta razón, se realizan estudios de contacto en esas instituciones cuando se han producido estos casos, y la incertidumbre de haber contraído la enfermedad puede alimentar temores. La información a la comunidad por parte del equipo de salud desde diferentes áreas es clave en ese momento para desmitificar esta enfermedad y minimizar las consecuencias de la estigmatización de los niños y adolescentes enfermos. ${ }^{17}$

\section{TABLA 1. Estudio de contactos}

- Identificación de cada uno de los convivientes y otros contactos que hayan permanecido más de 6 horas diarias con el enfermo de tuberculosis.

- Evaluación clínica, prueba tuberculínica (purified protein derivative, PPD) y radiografía de tórax a todos los niños y adolescentes.

- Evaluación clínica y radiografía de tórax a los adultos.

- Baciloscopía de esputo a los adolescentes y adultos sintomáticos respiratorios (tos y expectoración de 15 o más días de evolución).
Tuberculosis también es sinónimo de tratamiento regular y prolongado, requisito indispensable para su curación. Es necesario que la persona enferma adhiera a este, entendiendo como adherencia el sostenimiento sistemático e ininterrumpido de las condiciones de medicación, cuidados y hábitos de vida indicados para el tratamiento. Se trabaja desde la educación para la salud y se estimula la participación de los pacientes y familiares para esclarecer todas sus dudas y temores, con un fuerte contenido informativo. ${ }^{6}$

Desde esa particularidad o singularidad del niño/joven y su entorno, se entiende la intervención del trabajador social, acompañando, conteniendo e informando, ya sea durante la internación o en la consulta ambulatoria. ${ }^{18}$

Cabe destacar que, en este proceso de adherencia, el rol del Estado es fundamental, no solo en la provisión gratuita de los medicamentos, que debe ser garantizada a nivel nacional, sino también en facilitar los recursos necesarios para que el paciente acceda a su atención y tratamiento, así como a los subsidios, pase libre de transporte, alimentación, mejora de la vivienda, etc., teniendo en cuenta que es una enfermedad de varios meses de tratamiento, que compromete la vida laboral y social de la familia, y afecta la calidad de vida de los niños. ${ }^{10,12,13}$

Desde lo institucional, se debe trabajar la noción de accesibilidad como elemento facilitador de la adherencia y como forma de efectivizar el derecho a la atención de la salud. Se hace referencia a la accesibilidad geográfica, a recibir en tiempo y forma la medicación, a la ampliación de turnos y horarios para acceder a los controles médicos y a lo que desde la especificidad como trabajador social adquiere especial relevancia: la accesibilidad que se logra a partir de la construcción de un vínculo de confianza y respeto que permita el intercambio de saberes y la reflexión conjunta en aras de que el adolescente se apropie del cuidado y la atención de su salud, y los adultos encargados del cuidado de los niños enfermos colaboren firmemente en el tratamiento. ${ }^{6}$

La tuberculosis suele ser comparada con la punta de un iceberg: ${ }^{19}$ al explorar lo que subyace, aparecen pobreza, hacinamiento, viviendas y lugares de trabajo mal ventilados, limitado acceso a servicios sanitarios, contaminación ambiental intradomiciliaria, VIH/sida, adicciones, falta de escolaridad, falta de controles 
de salud de embarazadas y niños. Identificar los determinantes sociales y los factores que favorecen la transmisión y el riesgo de enfermar de tuberculosis (Gráfico 1) es acceder a puntos que requieren de atención y oportunidades de intervención para disminuir su prevalencia y, fundamentalmente, disminuir la morbimortalidad por tuberculosis en las poblaciones más vulnerables. ${ }^{7,10,16}$

\section{Objetivo general de la intervención del trabajador social}

Disminuir el riesgo de infectarse, enfermar y morir por tuberculosis, mediante la implementación de acciones de promoción, prevención, atención y rehabilitación de la salud.

\section{Objetivos específicos}

- Contribuir a la visibilidad de la tuberculosis pediátrica como enfermedad prevalente.

- Favorecer la realización de actividades comunitarias de información y prevención.

- Abordar en forma integral las problemáticas de niños y adolescentes con tuberculosis y sus redes sociofamiliares.

- Facilitar el acceso a la atención, mediante la coordinación y articulación con otros servicios e instituciones.

- Fomentar el abordaje interdisciplinario de la temática tuberculosis pediátrica.

- Aportar al equipo de salud la perspectiva social del proceso salud-enfermedadatención-cuidado, accesibilidad ampliada y adherencia al tratamiento de niños y adolescentes.

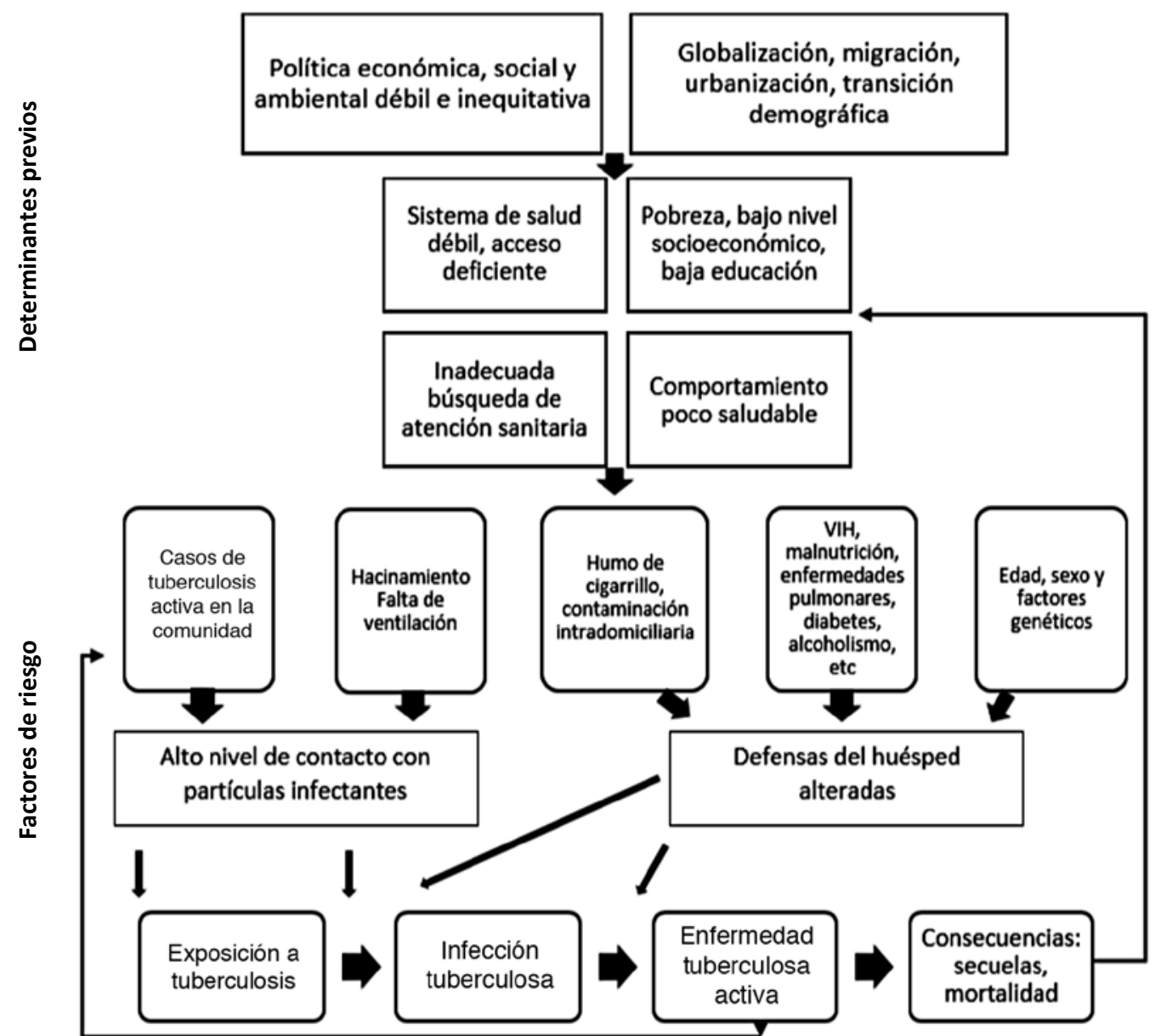

Modificado de referencias 7 y 10.

VIH: virus de inmunodeficiencia humana. 
- Promover la participación en actividades de docencia e investigación.

\section{Prioridades de intervención}

Tomando el abordaje de salud integral, y teniendo en cuenta las situaciones especiales de mayor riesgo de morbimortalidad, ${ }^{20}$ se podrán establecer las siguientes prioridades desde el diagnóstico clínico social:

- Tuberculosis con resistencia a fármacos.

- Menores de 5 años.

- Adolescentes.

- Coinfección con VIH/sida.

- Meningitis tuberculosa.

- Tuberculosis pulmonar con bacteriología positiva (bacilíferos).

- Tuberculosis extrapulmonar.

- Problemáticas sociales complejas.

\section{Actividades luego del diagnóstico de} tuberculosis

- Realizar entrevistas para establecer una relación personalizada con el paciente y su familia a fin de conocer datos de su historia de vida, informar sobre las características de la enfermedad, su tratamiento y la importancia del control de salud de los contactos. Ofrecer un espacio de contención y reflexión sobre el impacto del diagnóstico y de escucha en relación con la expresión de dudas, angustias, temores, mitos y preconceptos.

- Evaluar la facilidad de acceso al lugar de atención y participar en la decisión de la modalidad y lugar de tratamiento a fin de favorecer la accesibilidad y la adherencia, y promover el ejercicio del derecho a la salud integral de los niños y adolescentes.

\section{Actividades durante el tratamiento}

- Identificar facilitadores y obstaculizadores para la adherencia al tratamiento, teniendo en cuenta los procesos de salud-enfermedadatención-cuidado del niño/joven y su familia.

- Sostener un vínculo de contacto y contención ante dificultades en la adherencia al tratamiento: realización de llamados telefónicos, citaciones por correo, entrevistas domiciliarias y/o comunicación con centros de salud $\mathrm{u}$ hospitales del área de residencia del paciente.

- Orientar y favorecer el acceso a la realización de los estudios de contactos (tabla 1) a fin de identificar el caso índice, otros adultos enfermos y / o detectar otros niños o adolescentes infectados o enfermos de tuberculosis.

- Intervenir en los casos institucionales (escuelas, comedores, hogares de niños, guarderías) y facilitar la comunicación entre profesionales para llevar a cabo el estudio y seguimiento de los contactos.

- Articular la derivación dentro del sistema a través de la red de efectores específicos para la atención de la tuberculosis según el lugar de residencia.

- Brindar orientación en las gestiones necesarias para la obtención de ayuda económica y/o social en caso de ser necesario.

\section{Actividades al finalizar el tratamiento}

- Fortalecer la percepción del cuidado de la salud en general y de los factores que pueden incidir en la persistencia de la tuberculosis en el grupo familiar y sus allegados, como la nutrición, los controles materno-infantiles, la prevención de adicciones y la consulta precoz ante enfermedades del aparato respiratorio.

\section{Actividades en todo momento}

- Actividades de prevención de la salud dirigidas a la comunidad en general y a la población pediátrica en particular con el propósito de brindar información acerca de esta enfermedad y evitar la estigmatización del paciente, lo que favorece la promoción de la salud y la adherencia al tratamiento.

- Reuniones con el equipo de salud interviniente para analizar las estrategias de intervención adoptadas, evaluar los resultados del tratamiento (curación, abandono, muerte) y recuperar pacientes perdidos en el seguimiento, aportando la perspectiva del diagnóstico social y estableciendo nuevas líneas de acción.

\section{CONCLUSIONES}

La tuberculosis en niños y adolescentes es consecuencia directa de la exposición a enfermos contagiantes en el medio donde viven y se desarrollan. El control de la tuberculosis depende de la compleja interacción de factores biológicos, políticos, sociales, económicos, culturales y ambientales. No puede separarse a los niños y adolescentes de su entorno para protegerlos del riesgo de enfermar y morir de tuberculosis. Es necesario emplear todos los recursos disponibles para hacer más efectivas las medidas de control de esta enfermedad; el aporte del trabajador social 
es uno de los más importantes para lograrlo.

$\mathrm{Al}$ abordar en forma integral las problemáticas de los niños y adolescentes con tuberculosis y sus redes sociofamiliares, deben priorizarse las situaciones de mayor riesgo de morbimortalidad y de falta de adherencia al tratamiento: niños pequeños, adolescentes, tuberculosis grave, VIH/ sida, problemas sociales complejos.

Se puede intervenir luego del diagnóstico, ofreciendo información y contención de los adolescentes y los padres o familiares a cargo de lactantes y niños pequeños. Durante el tratamiento, brindando orientación para favorecer la accesibilidad y adherencia a este y al control de los contactos de mayor riesgo de infectarse y enfermar de tuberculosis. Se puede involucrar a la comunidad, informar, evitar la estigmatización del niño enfermo y su familia y favorecer la promoción de la salud.

\section{Agradecimientos}

A la Red de Trabajadoras Sociales que intervienen en Tuberculosis del área metropolitana de Buenos Aires.

\section{REFERENCIAS}

1. World Health Organization. Global tuberculosis report 2015. 20th ed. Geneva: WHO, 2015. [Acceso: 26 de noviembre de 2016]. Disponible en: http: / / apps.who.int / iris / bitstream/10665/191102/1/9789241565059_eng.pdf.

2. Notificación de Casos de Tuberculosis en la República Argentina Período 1980-2015. PRO.TB.DOC.TEC. N. ${ }^{\circ}$ 29/16. Santa Fe: INER-ANLIS-MSAL, 2016. [Acceso: 8 de abril de 2017]. Disponible en: http:/ / www.anlis.gov. ar/iner/wp-content/uploads / 2016/11/ Notificacionde-casos-de-Tuberculosis-en-la-Republica-ArgentinaPeriodo-1980-2015.pdf.

3. Mortalidad por Tuberculosis en menores de 20 años en Argentina 1980-2015. PRO.TB.DOC.TEC.11/17. Santa Fe: INER-ANLIS-MSAL, 2017. [Acceso: 8 de abril de 2017]. Disponible en: http:/ / www.anlis.gov.ar/iner/wpcontent/ uploads / 2016/04/Mortalidad-por-Tuberculosisen-menores-de-20-años-en-Argentina-1980-2015.pdf.

4. Goosby E. Out of the shadows: shining a light on children with tuberculosis. Int J Tuberc Lung Dis 2015;19(Suppl1):S1-2.

5. Bossio JC, Arias SJ, Fernandez HR. Tuberculosis en Argentina: desigualdad social y de género. Salud Colectiva 2012;8(Suppl 1):S77-91.
6. Castilla MV, Ferrari Mango C. Tuberculosis y lógicas de cuidado en barrios marginales y vulnerables de Buenos Aires, Argentina. Rev Pilquen Secc Cienc Soc 2015;18(3):36-49.

7. Lönnroth K, Jaramillo E, Williams BG, et al. Drivers of tuberculosis epidemics: The role of risk factors and social determinants. Soc Sci Med 2009;68(12):2240-6.

8. Saunders MJ, Evans CA. Fighting poverty to prevent tuberculosis. Lancet Infect Dis 2016;16(4):395-6.

9. Fuentes-Tafur LA, Ticona Chávez E, Velasco Guerrero JC, et al. El Plan TBCero: Un enfoque integral para el control de la Tuberculosis. Acta Med Per 2012;29(2):104-12.

10. LönnrothK, JaramilloE, Williams BG, et al Tuberculosis: the role of risk factors and social determinants. In Blas E, Kurup S, edit. Equity, social determinants and publichealth programmes. Geneva: World Health Organization, 2010:219-42. [Acceso: 26 de enero de 2017]. Disponible en: http: / / apps.who.int / iris/bitstream/10665/44289/1/9789241563970_eng.pdf.

11. Grange JM, Gandy M, Farmer P, et al. Historical declines in tuberculosis: nature, nurture and the biosocial model. Int J Tuberc Lung Dis 2001;5(3):208-12.

12. Arango Loboguerrero M. Importancia e impacto social de la tuberculosis en los niños. Medicina (Bogotá) 2015;37(3): 286-93.

13. Williams G, Alarcón E, JittimaneeS, et al. Prácticas óptimas en la atención a los pacientes con tuberculosis. Una guía destinada a los países de bajos ingresos. París: UICTER, 2007. [Acceso: 23 de mayo de 2017]. Disponible en: http: / / www.sld.cu/galerias/pdf/sitios/tuberculosis/union_ bestpractice_spanish_web.pdf.

14. Abad Campaña P, Cortés Gimeno V. La tuberculosis, un reto para el trabajador social. Trab Soc y Salud 1999;32:397-411.

15. Moro Gutierrez L. Condicionantes sociales y culturales en la vivencia de la tuberculosis. Trab Soc y Salud 1999;33:369-82.

16. Craig GM, Daftary A, Engel N, et al. Tuberculosis stigma as a social determinant of health: a systematic mapping review of research in low incidence countries. Int J Infect Dis 2016;56:90-100.

17. García Campos D, Llordes Llordes M, Urgelles Homdedeu $\mathrm{M}$, et al. Intervención social en un brote de tuberculosis en una escuela de primaria. Trab Soc y salud 2004;47:327-30.

18. Fernández Fernández MR, Peñafiel Escamez MT, Gutierrez C, et al. Justificación de la presencia de una Trabajadora Social en una Unidad de Tuberculosis. Trab Soc y Salud 2004;47:313-9.

19. Lorenzo RubioJL, González Martín A, Rodríguez González A, et al. Tuberculosis un problema social. Gac Med Espirit 2002; 4(1). [Acceso: 28 ene 2017]. Disponible en: http:/ / bvs.sld.cu/revistas/gme/pub/vol.4.(1)_06/vol.4.1.06.pdf.

20. Comité Nacional de Neumonología e Infectología de la Sociedad Argentina de Pediatría. Criterios de diagnóstico y tratamiento de la tuberculosis infantil. Resumen ejecutivo. Arch Argent Pediatr 2016;114(2):189-190. 